\title{
Clandestins au secret. Contrôle et circulation de l'information dans les centres de rétention administrative français
}

Nicolas Fischer

\section{(2) OpenEdition}

Journals

Édition électronique

URL : http://journals.openedition.org/conflits/1739

DOI : $10.4000 /$ conflits. 1739

ISSN : $1777-5345$

Éditeur :

CCLS - Centre d'études sur les conflits lilberté et sécurité, L'Harmattan

\section{Édition imprimée}

Date de publication : 1 mars 2005

Pagination : 91-118

ISBN : 2-7475-8103-9

ISSN : 1157-996X

\section{Référence électronique}

Nicolas Fischer, "Clandestins au secret. Contrôle et circulation de l'information dans les centres de rétention administrative français », Cultures \& Conflits [En ligne], 57 | printemps 2005, mis en ligne le 06 janvier 2010, consulté le 30 mars 2021. URL : http://journals.openedition.org/conflits/1739; DOI : https://doi.org/10.4000/conflits.1739

Ce document a été généré automatiquement le 30 mars 2021.

Creative Commons License 


\title{
Clandestins au secret. Contrôle et circulation de l'information dans les centres de rétention administrative français
}

\author{
Nicolas Fischer
}

1 La multiplication contemporaine des lieux d'internement dans l'espace européen et leur diversification, marque pour de nombreux auteurs le passage progressif d'une politique d'expulsion des étrangers présents irrégulièrement sur le territoire, à une politique "pro-active» tendantplutôt à empêcher les migrants d'y pénétrer ${ }^{1}$. Conséquences et symptômes de cette évolution, les espaces d'internement tendent à se délocaliser hors de l'espace Schengen, constituant une ceinture de camps entourant ses limites. Cette nouvelle logique ban-optique, mettant moins l'accent sur une surveillance-redressement que sur la mise à l'écart de la population, accentue alors la distance entre le corps du citoyen démocratique occidental et son symétrique opposé, celui de l'interné dépossédé de son existence juridique et à bien des égards de toute existence sociale ${ }^{2}$. Ce double effacement de l'individu hors du monde humain entendu comme ordre des interactions ordinaires, se renforce alors d'un effacement du lieu même de l'enfermement. Proprement "escamoté » de l'espace public et de toute possibilité de contrôle démocratique, le camp d'internement contemporain est fréquemment un camp qui « n'existe pas $»^{3}$.

2 Dans ce contexte, on tentera ici d'envisager un autre type de camps, mis en réseau avec ces espaces et ces corps invisibles des frontières de l'Europe mais contrastant fortement avec eux: les centres de rétention administrative utilisés en France pour l'enfermement des étrangers en instance d'éloignement du territoire. Ces centres, qui possèdent leur équivalent dans la plupart des Etats de l'espace Schengen, ont la particularité de se trouver directement sur leur territoire, et de relever encore pour l'essentiel de leurs droits internes. 
3 Cette dimension « intérieure » les situe au contact immédiat des sociétés européennes, avec un effet doublement problématique. La rétention s'insère tout d'abord dans le dispositif d'éloignement du territoire, comme c'est le cas en France depuis 1981. Mais elle ne peut dès lors intervenir que pour des étrangers déjà présents, et en outre déjà arrêtés et déjà frappés d'une mesure d'éloignement. Elle est de fait prévue pour les cas d'expulsion administrative ou judiciaire des étrangers délinquants, et surtout de reconduite à la frontière ou de réadmission des étrangers en situation irrégulière (les « sans-papiers » les plus fréquemment visés).

4 La rétention doit alors être replacée au sein d'un dispositif plus vaste visant à gérer la population des «irréguliers ». Elle est inséparable, à ce titre, de la dialectique du secret et de la détection qui marque leur rapport à l'Etat. Pour l'étranger "sans-papiers ", l'enjeu est de cacher au public la réalité de sa situation. A l'inverse, les administrations des Etats occidentaux multiplient les dispositifs de surveillance visant à dévoiler et inscrire sa présence physique sur le territoire, construisant moins une «Europe forteresse » qu'une «Europe panoptique » ${ }^{4}$. A l'instar d'autres dispositifs de gestion du risque, ces techniques envisagent les sans-papiers - volontiers associés à un hypothétique "risque migratoire " - comme une présence diffuse et insaisissable qu'elles doivent permettre de convertir en information traitable.La rétention est alors la technique ultime de cette surveillance, pour des étrangers déjà détectés, qu'il s'agit de regrouper sur un territoire délimité pour mieux accomplir leur «éloignement effectif $»$. Il vise lui aussi à rendre visible une présence produite comme clandestine et indésirable, afin d'acquérir sur elle des "prises» qui la rendront plus aisément manipulable ${ }^{5}$ : en l'occurrence, il s'agit de saisir ces individus par hypothèse incontrôlables pour les maintenir dans un espace clos, et préparer plus facilement leur éloignement.

5 C'est cette logique particulière de jeu sur l'information disponible qui donne aux centres de rétention leur forme comme institutions «totales », et délimite une grande partie des rapports de forces qui s'y déploient. Comme lieux d'enfermement et de prise en charge d'un matériau humain ${ }^{6}$, ils assurent une "clôture sociale $»^{7}$, arrachant l'étranger aux interactions auxquelles il prenait part précédemment, et le dépossédant du contrôle qu'il pouvait exercer sur l'information dans ses rapports à autrui. Pour autant, la «mortification» impliquée par cette dépossession des rôles sociaux antérieurs ne peut revêtir le caractère absolu que l'on vient de noter pour les camps de la périphérie de l'espace Schengen. Ainsi que le rappelle Johanna Siméant, l'étranger, même sans-papiers et reconductible à la frontière, s'inscrit peu ou prou dans un « réseau d'interdépendances élargies » dès lors qu'il est placé au contact des espaces publics des démocraties occidentales ${ }^{8}$. La clôture des interactions ne peut donc se faire à "sens unique ", du sans-papiers vers l'administration. La puissance de l'Etat ne s'y affirmera pas par la mise en œuvre d'une rupture sociale radicale entre l'étranger enfermé et l'extérieur, avec pour résultat sa disparition pure et simple. Pour gouverner les corps et les agencer au mieux, il sera plutôt nécessaire de gérer et moduler les liens multiples qu'ils conserveront et créeront parfois avec l'espace public depuis le lieu même de la rétention. Non pas coercition pure et simple donc, mais « droite disposition des choses " dans les termes de Michel Foucault ${ }^{9}$ : la bonne gestion de la rétention administrative passera donc par le déploiement d'une logique complexe de prélèvement, de « fuite » et divulgation de l'information entre les multiples acteurs qui la font exister. 
6 C'est cette dynamique que nous voudrions élucider, sans avoir toutefois la prétention de la restituer intégralement dans sa complexité. On reviendra tout d'abord sur la définition même du centre de rétention comme espace fermé au public, et sur les difficultés posées par la création de cette fermeture inédite - l'étude de la genèse des centres sera ici particulièrement éclairante. On envisagera ensuite la manière dont la définition minimale des échanges légitimes d'information en rétention se trouve subvertie au quotidien sous la pression indirecte de ce même espace public.Le travail de la Cimade, qui intervient depuis 1984 dans les centres de rétention français et constitue un lien essentiel avec "l'extérieur", devra ici faire l'objet d'une attention particulière $e^{10}$.

\section{La délimitation du centre comme arène clôturée et comme régime d'interaction}

\section{De la pratique policière au centre spécialisé}

7 Si l'étude de la genèse de la rétention est d'un réel intérêt, c'est aussi parce qu'elle débute justement avec un camp qui «n'existe pas », et se trouve dénoncé comme tel. Il s'agit du centre d'Arenc, installé dans un entrepôt désaffecté du port autonome de Marseille et utilisé par la police dès 1964 pour le maintien des étrangers en instance d'éloignement du territoire, alors que sa création et son fonctionnement ne sont prévus à l'époque par aucun texte ${ }^{11}$. De plus en plus sollicité après l'arrêt de l'immigration en 1974, le centre est "découvert» en avril 1975 par un journaliste de l'Humanité. Publiquement dévoilé, l'enfermement des étrangers suscite alors une mobilisation qui prend rapidement une dimension nationale et ne s'éteint qu'en 1979. On y retrouve significativement la dénonciation de l'opacité, qui domine à l'époque dans les mobilisations portant sur la condition juridique des étrangers en France ${ }^{12}$ : dans la presse et les textes militants, Arenc est une "prison clandestine", une "prison sauvage » ou un «camp d'internement secret ». Son existence n'est d'ailleurs connue qu'à la suite de disparitions d'étrangers dont leurs proches ignorent l'arrestation et $a$ fortiori le lieu et le motif de leur détention, mais qui « réapparaissent » pour témoigner sur les conditions d'enfermement dans l'entrepôt de Marseille ${ }^{13}$.

8 La mobilisation ne débouche pourtant pas sur l'interdiction de la rétention administrative, mais au contraire sur sa reconnaissance officielle, puis sa légalisation entre 1977 et 1981. Ce travail d'officialisation aboutit sous le gouvernement socialiste de P. Mauroy, lorsque la « loi Questiaux» du 29 novembre 1981 ajoute à l'ordonnance de 1945 l'article 35bis qui régit encore actuellement la rétention. Ce dernier reste cependant très elliptique. Les centres de rétention n'y sont définis que négativement, comme "locaux ne relevant pas de l'administration pénitentiaire "; les étrangers enfermés reçoivent quelques garanties - auxquelles s'ajouteront trois ans plus tard l'intervention de la Cimade - mais leur statut reste incertain.

9 Si l'opacité radicale du centre n'est plus tenable, le travail de définition du régime d'enfermement acceptable se fera donc en pratique, par la tentative de résolution d'une question essentielle : comment s'assurer d'une «prise » efficace sur la population des étrangers en instance d'éloignement? Ainsi posée, la problématique rappelle l'impératif de "gestion différentielle des illégalismes » évoquée par Foucault à propos de la prison pénale, et déjà rapprochée de la question de la rétention ${ }^{14}$. De fait, après le vote 
de la loi Questiaux, le cadrage progressif de la rétention comme mode de saisie spécifique des étrangers éloignés s'effectue à partir des locaux de police utilisés pour la surveillance des délinquants en général. Faute de cadre juridique précis, ces derniers continuent en effet à être utilisés. Une circulaire interne du ministre de l'Intérieur peut encore souligner en octobre 1983 que "à l'heure actuelle, les locaux utilisés à cette fin [le maintien des étrangers] sont principalement les salles de garde à vue des commissariats de police et ceux disponibles dans les aéroports et les ports maritimes "; «Il importe en tout état de cause de mettre fin à la détention des immigrés dans les locaux de garde à vue $»^{15}$.

Cette parenté originelle avec la garde à vue n'a rien d'anodin. Au final, la rétention, en effet, rompt moins avec sa logique qu'elle ne la recadre sur la personne de l'étranger reconduit à la frontière, et sur le prélèvement d'informations nécessaires à cette reconduite. A l'instar des différentes techniques de gestion de la délinquance, la rétention s'insère ainsi dans une série de "prises" dont la personne suspecte étranger comme délinquant - fera l'objet sur la voie publique. Chacune de ces prises correspond pour l'individu à une restriction du champ des interactions auxquelles il peut librement prendre part, et par conséquent à une perte plus ou moins radicale du contrôle sur l'information qu'elle diffuse à autrui sur elle-même ${ }^{16}$. Ainsi de l'arrestation sur la voie publique analysée par F. Jobard : brusquement arrachée à l'anonymat de l'espace public, la personne arrêtée voit soudain son monde se restreindre à une interaction unique avec les forces de l'ordre. L'examen du document d'identité qui l'accompagne ajoute à cette interaction une médiation essentielle en confirmant ou infirmant le soupçon policier sur la véritable «identité sociale» de la personne contrôlée ${ }^{17}$. Son usage "ferme» un peu plus l'interaction entre les policiers et leurs "clients", avant que la garde à vue confirme cette fermeture par une clôture physique $^{18}$.

11 La restriction de l'interaction libre et en «face à face » s'accompagne donc de la mise en place d'une relation exclusive avec les agents de l'Etat, médiatisée de façon croissante par le recours aux attestations écrites. Cette logique est d'autant plus accentuée lorsque c'est un étranger "clandestin » qu'il s'agit de saisir. L'absence de documents de séjour constitue pour lui un "schème directeur d'assignation statutaire " (master-status-determining-trait) ${ }^{19}$ qui surdétermine toutes les interactions auxquelles il prend part, et suppose un contrôle permanent de l'information. La vérité sur son statut est ainsi dévoilée sélectivement aux interlocuteurs avec lesquels il est parvenu à nouer une relation directe de proximité. A l'échelle locale d'un quartier ou d'un arrondissement, le «monde » du sans-papiers se fait ainsi de moins en moins secret, mais s'articule sur des réseaux d'interconnaissance où sa situation administrative est connue et acceptée au moins tacitement ${ }^{20}$. A l'opposé, G. Engbersen note la hantise des sans-papiers pour les traces écrites qu'ils pourraient laisser derrière eux : parce qu'il ne faut pas que l'attitude ou les confidences de l'étranger irrégulier amènent l'administration à produire une information sur lui, c'est-à-dire à détenir une trace de son existence, il doit se cantonner, précisément, à l'informel dont la vertu première est de n'en laisser aucune ${ }^{21}$.

Le contraste est alors d'autant plus fort avec l'arrestation de l'étranger et le passage à une relation organisée autour de l'inscription de sa présence et de la production de traces écrites officielles - autant de mises en forme ${ }^{22}$ de sa situation. Progressivement « saisi » par le dispositif d'éloignement du territoire, le sans-papiers passera ainsi par une série de techniques de contrôle mises en réseau qui l'isolent un peu plus à chaque 
étape des réseaux locaux d'interconnaissance qui lui permettaient de se maintenir sur le territoire. Ces techniques augmentent, à rebours, sa dépendance à l'égard des documents écrits, dans l'optique de sa reconduite à la frontière.

Censée ouvrir directement sur le départ physique de l'étranger, la rétention intervient de ce fait dans le moment ultime où cette double logique s'intensifie. Elle parachève alors le cadrage de la surveillance sur le contrôle spécifique de l'étranger « indésirable ». De même que le délinquant chez Foucault n'était jamais « libéré », mais réinclus dans une arène délinquante fermée sur elle-même, le centre de rétention réalise la fermeture de l'arène de "gestion différentielle des sans-papiers » dans laquelle il ne s'agit pas d'inclure le corps pour en réagencer les forces, mais de créer son exclusion pure et simple, tout au moins socialement à défaut d'éloignement physique ${ }^{23}$. C'est au centre, dans son fonctionnement concret, de parachever cette exclusion.

\section{Saisir les corps en saisissant l'information : la double clôture des centres de rétention}

\section{Discriminer entre immigrés et retenus}

La conception même de la rétention illustre ce cadrage et cette spécialisation des centres au regard de l'arène délinquante gérée par le carcéral. Les plans du ministère de l'Intérieur prévoient ainsi la création d'un réseau de camps permettant un quadrillage le plus étroit possible du territoire, et prenant en charge l'étranger dès qu'il cesse de relever du système pénal général. On prévoit ainsi « des locaux dans des points diffus $d u$ territoire afin d'assurer l'hébergement des immigrés immédiatement après la décision [de reconduite]» complétés par "l'implantation de constructions modulaires dans les cinq principaux aéroports de départ des immigrés ${ }^{24}$.

15 L'emploi du terme "immigré » souligne pourtant combien la tâche de distinction entre les futurs « retenus » et l'immigration de travail présente en France en général ne va pas de soi. C'est la définition même de la rétention qui effectuera ce travail de catégorisation et de séparation entre les retenus et les autres, tendant à produire comme groupe particulier ces étrangers absolus que sont les expulsables ${ }^{25}$ - comme en témoigne cette réserve du ministre des Transports sur "L'implantation des centres d'accueil dans les aéroports (...) les personnes faisant l'objet d'une procédure de reconduite à la frontière seraient en contact avec le personnel des entreprises sous-traitantes des aéroports composés en majeure partie de travailleurs immigrés (...) il conviendra de trouver une solution afin que les immigrés ne soient pas en contact avec [ce] personnel $»^{26}$.

Ce qui risque par excellence d'enrayer la logique de spécialisation du centre, c'est précisément ce mélange entre travailleurs immigrés pas encore ou pas assez séparés, c'est cette interaction non contrôlée qui mettrait en présence des « retenus » et des employés « légaux » de l'aéroport devenus subitement indistincts, jugés suffisamment proches pour se mêler, et peut-être susciter un mouvement collectif qu'il serait tout aussi impossible de contenir. La première mise en œuvre d'un contrôle sur les corps, c'est donc cette clôture physique entre l'espace public qui environne immédiatement le centre, et les "retenus » qui sont produits comme tels par le mouvement même de fermeture. Mais cette fermeture trouve son complément dans une deuxième clôture du centre, dont le ressort est le prélèvement direct de l'information et l'inscription papier ou informatique - de sa présence. 


\section{La clôture " administrative » : comment faire de l'information un bien rare}

17 Cette seconde clôture " administrative » du centre apparaît clairement dans l'exemple $\mathrm{du}$ centre du Mesnil-Amelot - Roissy ${ }^{27}$. Elle constitue l'essentiel du travail des gendarmes assurant la garde du centre, et s'effectue presque intégralement dans le « local administratif » situé dans le bâtiment d'accueil au seuil du centre, à hauteur du premier réseau grillagé isolant l'espace de rétention de l'extérieur. En traversant cet espace par lequel doit nécessairement passer tout acteur qui pénètre ou quitte le lieu de rétention, l'étranger franchit donc la clôture séparant l'intérieur du centre de son extérieur. Mais il subit simultanément, dans les premières minutes de son entrée, les opérations fondamentales confiées au dispositif: notamment, le prélèvement d'informations qui parachève la " prise » dont l'administration cherche à s'assurer sur l'étranger en instance d'éloignement.

L'accomplissement de ces opérations s'effectue selon un ordre précis matérialisé dans l'organisation spatiale de l'accueil. Le bâtiment d'accueil est traversé par un couloir menant les étrangers et leur escorte de l'extérieur du centre à une seconde clôture qui permet de pénétrer dans l'espace de rétention proprement dit. Avant d'être autorisé avec son escorte à poursuivre sa route vers l'extrémité du couloir, un étranger qui entre au centre pour la première fois doit tout d'abord subir une fouille, puis s'entretenir avec un gendarme qui lui signifie sa situation et ses droits en rétention, et lui fait signer le procès verbal attestant qu'il en a pris connaissance. Cette première " dégradation ${ }^{28}$, qui lui signifie qu'il entre dans un espace où désormais le contrôle de son propre corps ne lui appartient plus, se complète par une "cérémonie d'admission » attestant sa transformation en retenu: c'est-à-dire son inscription dans l'espace de rétention, dûment confirmée par un document administratif qu'il est lui-même invité à signer.

19 C'est de même ce procès-verbal de début de rétention qui permet de passer à la seconde opération de saisie du corps. Une fois dressée en effet, cette première «trace » de la présence de l'étranger dans le centre est transmise aux gendarmes du local administratif, mais on lui ajoute un ensemble d'autres pièces communiquées par l'escorte : celles qui concernent plus directement la situation administrative de l'étranger, le motif de son placement en rétention (la nature de la mesure d'éloignement qui le concerne) et l'organisation de son départ. Sur ce dernier point, les fonctionnaires du local administratif se voient également transmettre les « documents officiels " saisis sur les étrangers au moment de la fouille, et qui sont «susceptibles de déterminer leur identité ou leur nationalité ${ }^{29}$ : il s'agit donc indifféremment de documents d'identité ou de passeports dont les gendarmes auraient fait la découverte. L'étranger qui traverse le local d'accueil n'est donc pas uniquement dépouillé des objets et possessions qui constituaient son "territoire du moi ». On prélève également sur lui des éléments qui, s'ils ne sont pas directement rattachés à son corps et ne le " dépouillent " pas physiquement, constituent des supports d'information essentiels.

L'importance particulière de ces supports (procès-verbaux, photocopies de décisions de justice et d'arrêtés préfectoraux, ou documents d'identité) apparait clairement dans l'usage qui en est fait dans le local de gendarmerie. Par l'information qu'ils comportent, ces documents administratifs permettent de rapporter directement la présence 
physique de l'étranger à son départ, en indiquant qu'il doit partir (décisions d'éloignement), et vers où il doit partir (passeports et documents consulaires). De fait, une fois transférées aux gendarmes de garde, ces données sont mises en équivalence avec l'aspect matériel de l'éloignement de l'étranger. On trouve ainsi sur un des murs du local un tableau à double entrée répertoriant le numéro de son procès-verbal d'entrée avec la date et l'heure de son arrivée, la nature et la référence de la mesure d'éloignement, la délivrance ou non d'un laisser-passer consulaire, l'horaire du vol prévu, et pour finir la date prévue de fin de la rétention ${ }^{30}$. Ces informations reproduisent elles-mêmes les données stockées sur le disque dur de l'ordinateur de la gendarmerie installé dans le local, et connecté aux réseaux et banques de données de la police de l'air et des frontières.

$\mathrm{Si}$ les étapes précédentes de la procédure d'éloignement dépossédaient progressivement le sans-papiers de tout contrôle sur l'information, les première minutes de rétention radicalisent donc le mouvement : le retenu cesse d'être considéré comme une présence physique, il est réduit à la seule information pertinente pour l'organisation de son départ. Bien plus : la maîtrise de cette information, inscrite tout entière et exclusivement sur le " papier ", prime sur la maîtrise de son corps. S'il s'agit non seulement d'identifier, mais surtout d'organiser le départ de l'étranger, l'attention des gardiens du centre se porte avant tout sur les documents attestant de sa nationalité - cas de la carte d'identité ou de son équivalent - ou de sa capacité à voyager - ce qui renvoie de façon privilégiée au passeport. La prise pertinente qu'il s'agit ici de saisir, c'est donc le lien juridique qui rattache l'individu à un Etat étranger (nationalité) ou qui le relie tout au moins à un Etat qui accepte de le reconnaître et de le protéger (passeport ou laisser-passer consulaire) ${ }^{31}$. La maitrise de ce lien constitue une prise nécessaire et suffisante permettant de rendre un phénomène jusqu'alors insaisissable les sans-papiers - plus « lisible », et par conséquent plus maniable - c'est à dire ici, expulsables.

22 Cette attention aux seuls documents qui permettront l'éloignement confirme le caractère ban-optique plus que panoptique du centre : il s'agit d'accomplir la stricte mise à l'écart de l'étranger, non d'analyser les faits et gestes des retenus, leurs traits corporels ou comportementaux. Le corps, ici, est même un obstacle, trop marqué d'incertitude là où les « identités de papier » constituent des attestations fiables ${ }^{32}$. Dans le prélèvement des documents administratifs que l'on vient de décrire, cette radicale mise à l'écart de l'étranger s'accomplit déjà : l'information inscrite sur les «papiers » se trouve totalement détachée de son corps, pour être transmise et exploitée dans des arènes extérieures au centre, mais cruciales pour l'éloignement : consulats, tribunaux ou bureaux des compagnies aériennes auprès desquels sont réservés les vols.

Il y a donc un extérieur administratif comme il y a un extérieur physique du centre, et la clôture par les papiers vient renforcer celle des barbelés. Instrument classique du contrôle à distance ${ }^{33}$, le document d'identité franchit même un stade supplémentaire dans la maniabilité en s'arrachant au support-papier d'origine pour être intégré aux fichiers informatisés nationaux et européens ${ }^{34}$. Le contrôle de l'information passe ainsi tout entier entre les mains de l'administration. Quant au retenu, il est symétriquement dépossédé de toute prise sur son destin. Avec le prélèvement de ses documents d'identité, la médiation de ses rapports avec autrui par les papiers se mue en dépossession: désormais dépourvu de pertinence et même dévalué en tant que 
pourvoyeur direct d'information, il passe du stade d'interlocuteur éventuel dans un interrogatoire à celui de simple corps à enfermer dans l'attente de son départ.

\section{Où le retenu devient un cadavre encombrant}

Tout autant que la clôture physique, l'information «séparée » du corps est donc un opérateur essentiel de la mortification à l'œuvre en rétention ${ }^{35}$. En quelque sorte, le principe même de fonctionnement du centre suppose l'absence d'attention au sort ultérieur de l'étranger. Après « l'accueil » en rétention, le retenu peut certes quitter le centre pour entrer dans un ensemble d'interactions : avec le juge de la rétention, le juge administratif qui instruit son éventuel recours, et, on l'a dit, les agents consulaires. Mais l'espace interne même de la rétention est dévolu à la seule attente, l'hébergement devant uniquement garantir la «maniabilité» minimale des corps, dont l'administration doit toujours pouvoir s'assurer le contrôle, ne serait-ce que pour les éloigner. L'ensemble de la disposition du centre peut donc n'être pensée qu'en fonction du simple hébergement d'un corps qui n'a, en quelque sorte, plus rien à dire, et dont on exige seulement qu'il persévère dans son être. Témoin de cette logique, la forme de l'hébergement : là où la cellule carcérale est pensée comme un révélateur panoptique et donc un pourvoyeur d'informations faisant du détenu un délinquant - l'habitation du retenu n'a pour but que de permettre sa subsistance corporelle. Les centres s'apparentent ainsi aux camps en général, fréquemment installés dans des lieux prévus pour des travailleurs, et constituant de ce fait moins un lieu de vie qu'un espace de reconstitution de la force de travail ${ }^{36}$. Ainsi de la première version du Mesnil-Amelot, installée de 1989 à 1995 dans des bâtiments de chantier ALGECO. Dans d'autres cas, les étrangers sont enfermés dans d'anciennes casernes de CRS (Nantes), ou des camps militaires dont certains ont du reste fréquemment servi à l'internement (Rivesaltes ou Vincennes).

La localisation des centres dans des bâtiments provisoires, baraques ou casernements, est cependant en contradiction avec l'oisiveté forcée des retenus, ainsi qu'avec la durée de leur présence dans le centre. Elle tend à accentuer les effets de la dépossession de soi qui vient d'être évoquée. Laissé « à l'abandon » parce que non pertinent comme corps physique, coupé de toute interaction directe avec autrui, le retenu est d'autant plus remis à un seul face à face avec des "forces sans visage " incarnées seulement par le "bâti et la durée »"

C'est pourtant ce corps "mortifié », radicalement exclu et arraché à l'ordre des interactions ordinaires, qui va échapper au contrôle des gardiens, se dérober à leur " prise » et parvenir à renouer, de différentes manières, une interaction non contrôlée avec l'extérieur. Alors qu'il semblait ne plus devoir faire l'objet de la moindre intervention, les gestionnaires du centre se verront donc forcés de lui manifester une attention particulière afin d'en recouvrer le contrôle. Mais quel type d'attention, pour quel type de contrôle? On vient de le dire, les points de fuite de la rétention par lesquels le corps échappe à ses gardiens sont multiples. Ces derniers devront ainsi, chaque fois, élaborer des réponses particulières, dont nous allons étudier deux exemples - deux cas qui supposent, chaque fois, de s'assurer une forme de maitrise de la circulation de l'information. 


\section{Sur quelques usages stratégiques de l'information en rétention}

\section{La souffrance publique du corps comme menace}

Dans un premier temps, l'élément incontrôlable n'est pas le seul corps meurtri du retenu, mais bien plutôt le lien qu'il parvient à tisser en tant que tel avec l'extérieur du centre. Si pour F. Jobard, l'automutilation constitue déjà une mise en échec du monopole étatique de la violence physique ${ }^{38}$, sa résurgence en rétention rejoint alors les remarques de J. Siméant sur l'atteinte au corps comme mode spécifique « d'irruption » des sans-papiers sur l'espace public. Le retenu apparait ici publiquement en tant que corps souffrant, mais c'est en tant que tel qu'il peut susciter une forme de soutien de la part de "l'opinion publique ", thématisée dans le registre humanitaire ${ }^{39}$. Même en tant que tel, il peut cependant constituer une menace pour la maitrise que les gardiens cherchent à conserver sur lui. Ainsi que le note un rapport dressé après un an de fonctionnement du centre du Mesnil-Amelot :

« (...) L'organisation actuelle du centre et (...) son apparence présente en font à tous égards une cible tout à fait symbolique et vulnérable, c'est du reste la raison pour laquelle depuis l'ouverture en juillet et jusqu'en décembre 1988, une manifestation de protestation a eu lieu tous les jeudis devant le centre. La triste apparence de l'ensemble permet des effets faciles aux photographes de presse (Cf. $\mathrm{n}^{\circ} 1386$ de l'hebdomadaire «Informations Ouvrières ", semaine du 28 septembre au 5 octobre 1988) et on devine le parti qui serait tiré d'un décès accidentel ou d'un suicide réussi dans le centre ${ }^{40}$.

Le corps du retenu est ici incontrôlable par excellence : c'est celui de la victime d'un accident, par hypothèse imprévisible, ou celui du suicidaire qui ne l'est guère plus. Mais la perte de contrôle provient surtout de l'existence d'un public susceptible d'assister à sa mutilation. Elle accompagne alors les marques publiques de sa nouvelle condition de corps enfermé - l'aspect extérieur du centre - et vient confirmer leur caractère dégradé. C'est la « survie » rudimentaire à laquelle se réduit l'existence des retenus qui suscite l'indignation publique. Le centre prend ainsi une dimension "symbolique", face à un public étendu formé par le lectorat potentiel d'une revue militante ou du travail d'un photographe. Si le contrôle du corps ne peut passer que par une clôture radicale de toute interaction, le corps ainsi arraché à l'espace des interactions ordinaires suscite donc encore une forme de solidarité précisément liée à son exposition publique comme corps mortifié. C'est le registre de l'humanitaire qui est alors mobilisé, réinsérant le corps souffrant du retenu dans un «réseau d'interdépendances élargi » suivant un schéma identique à celui des mobilisations de sans-papiers ${ }^{41}$.

Pour autant, ce nouveau lien tissé entre le public « extérieur » et le corps enfermé du retenu ne se limite pas au soutien abstrait de «l'opinion ». S'il constitue une menace réelle pour les fonctionnaires du centre, c'est qu'il se matérialise dans ses abords directs. Le public se réduit alors aux manifestants réunis chaque jeudi devant le centre, mais ce dernier cesse d'être uniquement symbolique. Il devient "vulnérable »: la dénonciation se convertit en agitation pouvant potentiellement mettre à mal le caractère « hermétique » de la clôture extérieure du centre. Les remarques qui suivent immédiatement l'extrait que nous venons de citer prolongent ce thème : 
« Un autre aspect de la sécurité concerne celle des gendarmes eux-mêmes ; outre la facilité avec laquelle des retenus pourraient se procurer des armes provenant de l'extérieur, voire organiser un mouvement collectif, l'état de santé de la population fréquentant le centre devrait constituer un grave sujet de préoccupation; le matériel de prophylaxie mis à la disposition des gendarmes et les précautions observées pour les fouilles semblent tout à fait dérisoires. Il a pu arriver que des gendarmes se blessent sur des lames de rasoir souillées présentes dans les bagages de retenus dont l'état de santé était douteux $»^{42}$.

La fouille ne permettant pas d'assurer suffisamment la stérilité du centre, ce dernier est constamment exposé au retour d'une interaction avec l'extérieur qui se donne à la fois pour immédiate et indétectable; c'est-à-dire au retour de l'informel qui marquait la vie clandestine de l'étranger. La "prophylaxie» doit alors garantir l'absence de contact avec l'extérieur, source de « contamination » : en premier lieu, l'introduction d'armes pouvant inverser les rôles entre gardiens armés et retenus normalement dépossédés de tout moyen de violence qui leur permettrait de se soustraire à leur contrôle ${ }^{43}$. La suite du rapport passe alors "naturellement " de cette contamination à une autre, plus directement sanitaire : la menace du VIH portée significativement par le corps même, mais aussi par le bagage qui l'accompagne, comme de tout élément venu du dehors et dont la maîtrise par les gendarmes reste insuffisante.

31 En d'autres termes, l'absence de maîtrise sur l'information publique portant sur la souffrance du corps constitue une menace aussi directe que l'absence de contrôle de sécurité à l'entrée des centres. Ne pas contrôler le régime d'énonciation dans lequel le corps du retenu sera publiquement thématisé, c'est en effet lui permettre d'entrer éventuellement dans des interactions non maîtrisées avec l'extérieur du centre, c'est mettre à mal la clôture de la rétention telle qu'on vient de l'envisager.

Pour les fonctionnaires chargés d'administrer les centres, il s'agira au contraire de recouvrer leur contrôle sur les corps. Faute d'abolir la solidarité dont le retenu fait l'objet, il faudra alors «l'étatiser »: si un lien se constitue avec l'extérieur à travers la compassion pour le corps, il faudra donc contrôler ce lien, se saisir de la manière dont il est thématisé, et du régime d'interaction du retenu avec autrui qu'il suppose éventuellement. Il s'agira également, par conséquent, de contrôler la relative ouverture du centre sur l'extérieur. La problématique du contrôle des corps se déplace alors vers celle du contrôle - incertain - des acteurs extérieurs chargés d'assister les retenus: c'est notamment le cas de la Cimade.

\section{La Cimade, ou le jeu difficile d'un « transporteur »}

3 D'une manière générale, la gestion minimale des corps retenus et de leur « survie » est en effet confiée à d'autres acteurs que les gendarmes ou les policiers. Au MesnilAmelot, cette division des tâches prolonge la division concernant l'information : aux gendarmes, la gestion de l'enregistrement des retenus, qui se borne comme on l'a vu au seuil de la rétention et à ses première minutes; à d'autres, en particulier les fonctionnaires du ministère de la Justice, le soin de gérer le «temps long » de l'attente et l'aspect matériel de l'enfermement, notamment le logement et la restauration des retenus. La gestion des centres de rétention s'est toutefois « enrichie » en une dizaine d'années d'autres intervenants dont le rôle s'inscrit cette fois directement dans l'attention au corps. C'est le sens de l'installation à partir des années 1990 de permanences médicales dans les centres. 
Les intervenants retrouvent ainsi spontanément la logique de l'urgence et du face à face direct propre à l'humanitaire ${ }^{48}$ : il s'agit de servir soi-même d'interlocuteur pour les étrangers que le placement en rétention isole radicalement, et de proposer un lien qui puisse limiter la dépossession à l'œuvre en rétention. Lorsqu'il s'agit par contre d'assurer les contacts avec l'extérieur, la Cimade reste cependant l'unique interlocuteur des retenus, leur permettant de renouer les relations rendues problématiques par l'enfermement tout en les contrôlant. Coupant court à toute relation informelle, ce régime particulier d'échanges officiellement cantonné à l'assistance humanitaire est réduit à une relation strictement médiate dans laquelle tout doit normalement passer par la Cimade. Surtout, cette relation est limitée aux rapports avec l'arène "clandestine» dont l'étranger a été séparé par son placement en rétention. Elle informe en premier lieu les familles sur le sort même de l'étranger ${ }^{49}$. Mais on pourrait multiplier les exemples des actions qui font des équipiers Cimade les véritables « voltigeurs » de la rétention, fréquemment amenés à quitter le centre sur demande des retenus pour des " missions » d'autant plus difficiles à accomplir qu'elles supposent un contact avec le monde semi-clandestin d'où ces derniers sont issus ${ }^{50}$.

Tenir ainsi la Cimade, c'est créer la possibilité d'une aide humanitaire au retenu, tout en cantonnant l'ampleur de cette solidarité dans les limites du rôle officiellement assigné 
à l'association. Ce dernier, qui amène la Cimade à effectuer les achats de dernière minute des étrangers retenus, ou encore à clôturer leurs comptes bancaires, est central dans les premières années de mise en pace des centres de rétention. Au début des années 2000, il est progressivement transféré aux fonctionnaires de l'office des Migrations Internationales (OMI), la mission de la Cimade étant requalifiée en accompagnement juridique - ce qui n'empêche pas les équipiers d'assurer ponctuellement cette assistance matérielle. De façon significative, il s'agit du seul registre d'action dans lequel l'intervention de l'association n'est aucunement problématique du point de vue de l'administration. En permettant de "liquider» définitivement les liens antérieurs du retenu avec les réseaux qu'il possédait, la Cimade reste en effet conforme au cadrage initial du centre comme espace de gestion et d'exclusion des corps. Lorsqu'en avril 1989 une première évaluation du fonctionnement des centres est tentée, le gestionnaire du Mesnil Amelot note ainsi que «l'action conduite par la Cimade" est "très utile. Pallie les inconvénients administratifs et s'occupe de la récupération des effets personnels $"^{51}$.

Mais l'intérêt du travail de la Cimade provient bien entendu des limites qu'elle impose à ce contrôle. Parce qu'ils restent extérieurs à l'espace de rétention, ses membres peuvent faire preuve d'une réelle distance au rôle institué. Dès son entrée dans les centres, l'association se réserve ainsi la possibilité d'un autre cadrage de l'information, dans lequel l'humanitaire redevient un régime public d'énonciation, utilisé dans le registre de la dénonciation. Les responsables du service de Défense des Etrangers Reconduits (DER) font valoir ce rôle dès 1984, en insistant sur leur «vocation de veille maintenant et, si les choses devaient se gâter plus tard, de témoin en vue de résister à l'inacceptable $\aleph^{52}$. On pourrait multiplier les citations dans lesquelles les membres de la Cimade s'écartent du rôle d'assistance en face à face supposé par la convention de 1984, pour affirmer également leur rôle de transporteur de l'information vers un extérieur, renvoyé cette fois à l'opinion et à l'espace public en général.

L'insistance sur le rôle de témoignage à l'extérieur tient sans aucun doute au positionnement simultané de l'association dans l'arène interne de la rétention, mais également à l'extérieur, devant le public restreint mais attentif des autres associations de défense des étrangers. Si les critiques lancées à l'encontre de la Cimade en 1984 se sont atténuées par la suite, sa présence dans l'arène de rétention reste cependant en butte à une potentielle suspicion de "collusion" avec l'administration, due à sa proximité avec les autorités gestionnaires des centres $^{53}$. De ce point de vue, la dénonciation publique permet à l'association de se distinguer justement des autres acteurs de la rétention, en s'affirmant aussi comme un acteur collectif au sein d'une arène publique où son rôle se définit dans la seule dynamique de la protestation - à l'opposé de sa fixation définitive par un texte ${ }^{54}$. L'action de la Cimade sur le terrain doit pour les équipiers eux-mêmes replacer les centres dans un lien à l'espace public : «pas simple service social, lieu de militance pour exercer des pressions à partir des abus constatés ${ }^{55}$.

41 Les équipiers reproduisent alors les classiques contradictions de l'exit, de la voice ou de la loyalty ${ }^{56}$, mais ce débat interne emporte un effet immédiat sur les relations de pouvoir à l'intérieur du centre. Dans la dynamique de la dénonciation, la publication de l'information sur la situation interne des centres renoue en effet avec l'exposition publique de la souffrance corporelle, c'est à dire du point de vue de l'administration avec une forme de perte de contrôle sur le corps. Pour les fonctionnaires, il faudra donc 
reprendre le contrôle de l'information et de l'acteur - collectif - qui en assure le transport.

La géométrie complexe de ce positionnement de la Cimade autour d'un jeu sur la diffusion publique de l'information est particulièrement illustrée par une "affaire " survenue à Lyon entre 1988 et 1990. Dans ce cas, il n'est même plus question de dénonciation, mais simplement de la possibilité d'une publicité immédiate du fonctionnement quotidien des centres: en décembre 1988, un journaliste de France Culture se voit refuser l'accès au centre de Lyon. Les équipiers Cimade protestent, arguant de l'impossibilité de toute dénonciation «humanitaire» de la rétention supposée par cette décision: "ce refus implique l'idée que l'opinion publique ne doit pas être informée du sujet $C R$ de manière directe après enquête d'un journaliste. (...) Or c'est justement pour éviter qu'il se passe des choses dans les commissariats de police et ailleurs que les CRA ont été légalisés et mis en ceuvre ${ }^{57}$. Significativement, cette dimension est directement interprétée comme une remise en cause du rôle même de l'association en rétention :

« La CIMADE est ainsi directement concernée par le refus opposé à ce journaliste et notre mission est d'obtenir (...) la possibilité qu'il y entre. Sinon notre collaboration avec les centres de rétention devient de la complicité avec ceux qui opposent le refus, empêchant le jeu normal de la démocratie, c'est-à-dire l'objectif de transparence des actions administratives de l'Etat - je pense que personne ne considère les $\mathrm{CR}$ comme secret ou raison d'Etat! (...) cela aurait le grand avantage de désamorcer les critiques qui sont faites à la CIMADE de gérer les CAMPS de CONCENTRATION modernes (...) ; j'ai entendu cette expression encore il y a trois semaines lors d'un congrès organisé par la Ligue des Droits de l'Homme. Mes explications et mises au point n'ont pas forcément convaincu du fait même de l'impossibilité d'entrer pour savoir $"^{58}$.

Face à une clôture totale du centre, les équipiers Cimade voient leur proximité à l'administration se transformer en complicité, et leur rôle se confondre avec celui des gardiens. Qu'il s'agisse de nuancer les dénonciations d'autrui ou de dénoncer soi-même, la proximité de l'association avec les gestionnaires des centres disqualifie son discours public. Ce n'est alors qu'en dénonçant l'opacité même du centre que ses responsables parviennent à la démarquer de l'arène interne de la rétention : cela passe par l'exigence d'ouverture du centre à la presse, mais le démarquage s'incarne également dans l'économie même de la présence de la Cimade en rétention: dans le même document, les responsables de la DER affirment que «la Cimade n'a pas lié sa parole, puisque la convention doit être renouvelée chaque année $\aleph^{59}$; de fait, lors du premier renouvellement en 1990, la direction de l'association envisage de cesser toute intervention dans les centres, en s'appuyant notamment sur les "rapports ambigus de la Cimade avec les ministères », et "l'interdiction à la presse d'accéder aux centres ».

La possibilité pour la Cimade de rompre la convention ou tout au moins d'en suspendre l'effet est centrale dans cette possibilité de « jeu » sur les rôles que ses militants sont à même de fournir. Accompagnée de communiqués et de déclarations publiques, elle fournit à l'association un moyen de pression plusieurs fois mis en œuvre par la suite, en réponse à l'aggravation constatée des conditions de rétention ${ }^{60}$. Dans ce cas cependant, le démarquage de l'association par rapport à l'administration et au rôle de simple assistance humanitaire que cette dernière avait défini pour elle prend la forme radicale d'un retrait pur et simple de l'association. Dans son intervention quotidienne dans les centres, le « jeu » demeure pourtant, et se fait permanent. Dans la mesure où la Cimade s'écarte de son rôle institué, sa place en rétention fait l'objet d'une négociation constante, dont l'usage que les équipiers feront de l'information est le pivot. Dans la 
mesure où les équipiers publient et dénoncent le traitement des retenus, ils se font les transporteurs d'une information précisément affranchie de tout contrôle, tout en définissant leur rôle en relation à une arène extérieure à celle de la rétention. Tout autant que le corps sur lequel elle informe, la Cimade échappe donc elle-même à la maitrise des fonctionnaires du centre, et devient à son tour suspecte de « clandestinité » ou de duplicité.

La direction de l'association est soucieuse de cette perte progressive de crédit liée aux dénonciations, et réaffirme la nécessité de rester au moins partiellement contrôlable par l'administration: "Certaines associations qui [ont dénoncé] trop systématiquement ne sont pas regardées comme crédibles par les pouvoirs publics. Nous pouvons être efficaces dans (...) nos rapports avec les gardes des centres, si nos interventions restent crédibles ${ }^{61}$.

\section{Le « mental » du retenu, ultime boîte noire}

C'est donc sur la Cimade, intervenant dans les centres tout en se réservant la liberté de les dénoncer, que se déplace l'enjeu du contrôle et la volonté de s'arroger des « prises ». La crédibilité que l'association doit chercher à préserver en est le ressort essentiel, tandis qu'elle se dérobe au contraire en rendant publique la situation en rétention. Ce rapport des forces renouvelé n'est cependant pas limité à la seule problématique de la dénonciation. Comme on l'a indiqué, il existe une clôture "administrative » au centre, plus immatérielle que sa fermeture physique mais tout aussi dégradante pour le retenu. Lorsqu'il s'agit pour lui de recouvrer la maîtrise de cette information bien particulière liée à l'organisation de son éloignement, la même logique de négociation sur son «transport» se déploie au cœur même du centre. Depuis les années 1990, elle s'est même trouvée accentuée par l'évolution du rôle institutionnel de la Cimade, qui inclut désormais la dispense d'une information juridique ${ }^{62}$. Dans ce cas, le nouveau rôle des équipiers, bien qu'institué, ne parvient pas à s'imposer en ce qu'il met à mal un aspect du contrôle du corps du retenu, celui qui doit s'exercer sur leurs affects, et ce que l'on finira par nommer leur « psychologie».

Là encore, nous prendrons l'exemple du Mesnil-Amelot et d'une crise qui s'y prolonge entre 1998 et 2002 autour du nouveau rôle de la Cimade. Il amène en effet les équipiers du centre à pénétrer chaque matin dans le local de gendarmerie afin d'y collecter une liste des retenus nouvellement entrés, et les indications devant permettre leur information; jusqu'à ce qu'une note interne de la gendarmerie vienne interdire aux intervenants extérieurs au centre la détention permanente d'un passe permettant d'entrer dans les bâtiments administratifs. Un responsable de la DER proteste alors en rappelant que depuis 1988, les intervenants de la Cimade disposent tous d'une habilitation personnelle qui justifie ensuite la délivrance de passes, mais une réunion organisée un an plus tard confirme le soupçon dont les équipiers sont l'objet: le libre accès de la Cimade au local est refusé, en raison de la confidentialité des informations gérées. On note également, un "manque de contacts réguliers et d'interlocuteur identifié » auprès duquel la mission exacte de la Cimade et les documents auxquels elle a accès puissent être précisés ${ }^{63}$.

48 Là encore, cette difficulté à pérenniser son rôle dans l'espace interne de la rétention marque un déficit de crédibilité de la Cimade. Son rôle de transporteur suppose ici une remise en cause de l'asymétrie d'information fondatrice opposant les gendarmes aux retenus, puisque les équipiers doivent à la fois en savoir autant que les gardiens, et 
pouvoir diffuser ce savoir auprès des étrangers enfermés. En 2001 encore, la Gendarmerie cherche à recouvrer sa position en s'appuyant sur une circulaire qui prévoit que la Cimade reçoive "les mêmes informations que les retenus " ${ }^{64}$. Niés dans leur rôle de transporteurs, les équipiers sont « confondus » avec la population enfermée et, de fait, s'ils ne donnent pas au retenu l'information qui lui rendra une prise sur son existence, ils peuvent être sans crainte assignés à une relation de proximité humanitaire avec elle.

Dans ce cas comme dans le précédent, l'enjeu reste le contrôle du corps du retenu, qui doit permettre d'exclure autant que possible les « points de fuite " par lesquels ce corps échappe à ses gardiens. Les protestations des responsables de la DER précisent ici la nature de cette fuite "corporelle", dans laquelle nous retrouvons la hantise de l'atteinte au corps: "Nous sommes conscients que la question des tentatives de suicide et des automutilations est un problème grave, rendu critique ces dernières semaines par la multiplication de ces faits. Pour autant nous ne pouvons accepter le règlement qui en est actuellement fait, visant implicitement à mettre en cause l'accès à l'information sur les départs pour les retenus ${ }^{65}$. "Nous nous permettons de signaler que les retenus ne comprennent en aucune manière la situation. Leur frustration par rapport au manque d'information laisse présager des réactions dont nous déclinons toute responsabilité $»^{66}$. Pour finir, le rapport de la Cimade pour l'année 2002 fait état du problème dans des termes identiques, en précisant toutefois que "bien au contraire, l'incertitude permanente est source de stress et de tension supplémentaire ${ }^{67}$.

Ressurgit donc le corps incontrôlable, potentiellement violent sur lui-même ou sur autrui. Mais la perte du contrôle n'est pas liée dans ce cas au lien avec l'extérieur. Presque symétriquement, elle est renvoyée à l'impact de l'information sur une « intériorité » que l'on prête au retenu. L'enjeu de la diffusion de l'information ici, c'est donc un état mental dont la portée est toutefois immédiatement corporelle : c'est la source des mutilations, des suicides et des révoltes par lesquelles le retenu voudra se soustraire au pouvoir.

51 Le flou dans lequel demeure la définition de cet «état » en fait un objet d'affrontement entre la gendarmerie et la Cimade. L'enjeu est la réaction supposée du retenu face à la dispense ou à la rétention de l'information sur les départs : l'information donnée étant une prise que le retenu arrache à ses gardiens pour se l'approprier, cette prise permetelle de l'apaiser, ou renforce-t-elle au contraire sa dépossession? Dans quel cas le retenu sera-t-il incité à une "évasion " ultime hors du contrôle par la tentative de suicide? La conclusion de cette " affaire » signe la contradiction ultime de l'espace de rétention. Dès 2000 en effet, une tentative de règlement instaure " une procédure d'alerte entre les différents services : lorsqu'un retenu fait apparaitre par son comportement des risques sérieux d'automutilation ou de tentative de suicide, une concertation (...) permet d'évaluer le type d'information qui peut lui être délivré (...) ${ }^{68}$. De façon significative, le dispositif est par la suite étendu à l'ensemble des centres par une modification introduite dans l'article 35bis par la loi Sarkozydu 26 novembre 2003 : « Sauf (...) si la personne ne parait pas psychologiquement à même de recevoir ces informations, l'étranger est informé par le responsable du lieu de rétention de toutes les prévisions de déplacement le concernant ${ }^{69}$.

"L'état psychologique » du retenu est donc un ultime point de fuite, interne au centre cette fois, et où les différents acteurs du centre se heurtent à un corps définitivement muet et insaisissable. Il désigne aussi, simultanément, la dimension de "l'intériorité " $\mathrm{du}$ retenu que la direction du centre devra autant que possible expertiser, 
diagnostiquer et prévoir pour recouvrer sa maîtrise sur les corps ${ }^{70}$. Si cette maîtrise peut alors passer par le contrôle de l'information, elle est cependant plus incertaine encore que lorsqu'il s'agissait de contrôler la dénonciation publique. Dans ce dernier cas, l'enjeu du contrôle était l'information elle-même, et le régime d'énonciation dans lequel elle devait être diffusée. Ici, l'information n'a pas pour effet de thématiser sous une certaine forme la souffrance du corps - elle fournit simplement au retenu les moyens de décider lui-même du contrôle, et de s'en soustraire ou non. En quelque sorte, il reste le seul maitre du jeu sur l'information. «L'état intérieur » qu'on lui prête est le lieu ultime de perception et de thématisation du corps souffrant, où le retenu reste seul à juger et évaluer la dépossession dont il fait l'objet, pour agir ensuite en conséquence. L'usage de l'information échappe alors nécessairement aux gardiens du centre: de fait, aucun règlement ne parvient à organiser de réelle saisie de cette dimension psychologique de l'étranger enfermé. Dans la loi de 2003, les «services » sont condamnés à négocier et évaluer chaque situation, en modulant notamment la diffusion des informations par la Cimade ou les forces de l'ordre. Ici comme dans le cas de la visibilité publique des corps, le contrôle de l'information par l'administration poursuit un corps qui ne cesse pas de se dérober.

Dans le cas de la rétention, les effets sociaux de l'enfermement et du souci humanitaire dont les fonctionnaires du centre ne peuvent faire l'économie semblent donc se conjuguer pour susciter des dispositifs de contrôle sans cesse plus étroits d'un corps qui pourtant, n'en finit pas d'échapper à la prise de ses gardiens. Parce qu'il est sommé de respecter les corps retenus, mais simultanément de les contrôler le plus étroitement possible, le centre de rétention est donc pris entre ces deux infinis de la gestion des corps, dont le contrôle de l'information est le pivot : ou bien, la diffusion de l'image du corps vers un espace public "humanitaire " potentiellement élargi au-delà même de l'espace national, ou bien le retrait de ce corps en lui-même, dans une réflexivité et une réappropriation de soi qui, de même, échappent à l'Etat.

Il devient alors nécessaire d'élaborer des dispositifs visant à gérer les nouveaux risques suscités par la logique même de la rétention ${ }^{71}$. Dans les deux cas que nous avons envisagés, cette gestion n'abolit jamais totalement la fuite - on ne peut empêcher l'information de "filtrer "; on ne peut dicter les réactions du retenu - et les prises que l'on cherche à acquérir ne sont donc jamais décisives. Au contraire : elles instituent officiellement l'humanitaire comme problématique centrale de la rétention, en faisant de la Cimade son gestionnaire officiel; de même, elles créent la "psychologie » du retenu comme intériorité insaisissable, et l'inscrivent dans la loi comme un élément durablement problématique, à évaluer au cas par cas. On retrouve ici la leçon de Michel Foucault : créer une relation de pouvoir nouvelle revient à créer simultanément les moyens de sa subversion. Si la réalisation "parfaite» du programme de la rétention comme gestion des corps ne saurait donc venir à bout des limites qu'il s'est lui-même données, le durcissement actuel de l'enfermement en France risque, par contre, de repousser le jeu de l'information et de la dégradation du corps vers des limites de plus en plus dramatiques. 


\section{NOTES}

1. Sur ce point, voir par exemple Guiraudon V., « De-nationalizing migration control. Analyzing state responses to constraints on migration control », in Guiraudon V. et Joppke C., Controlling a new migration world, Routledge, London et New York, 2001.

2. Sur cette mise à distance, voir Bigo D., Polices en réseaux : l'expérience européenne, Paris, Presses de Sciences Po, 1996 et Bigo D., "The globalisation of (in)security », Traces, Sovereign Police, Global Complicity, $\mathrm{n}^{\circ} 3 / 2003$; également Brossat A., La démocratie immunitaire, Paris, La Dispute, 2004.

3. Sur les camps en général voir Caloz-Tschopp M-C., Les étrangers aux frontières de l'Europe et le spectre des camps, Paris, La Dispute, 2004.

4. Engbersen G., "The Unanticipated Consequences of Panopticon Europe », in Guiraudon V. et Joppke C., Controlling a new migration world, op. cit., pp. 222-246.

5. Pour une analyse similaire des dispositifs de lutte contre le terrorisme, voir Linhardt D., "L'économie du soupçon. Une contribution pragmatique à la sociologie de la menace ", Genèses, $\mathrm{n}^{\circ} 44$, septembre 2001, pp. 76-98. En quelque sorte, la logique de regroupement du centre relève de la quarantaine, mais se trouve mise en réseau avec un dispositif de surveillance qui relève clairement quant à lui de la traçabilité. Voir Torny D., «La traçabilité comme technique de gouvernement des hommes et des choses ", Politix, n44, 1998, pp. 51-75.

6. Selon les termes de Goffman E., Asiles. Essai sur la condition sociale des malades mentaux et autres reclus, Paris, Minuit, 1968.

7. Selon le mot de Leca J., « Nationalité et citoyenneté dans l'Europe des immigrations », in CostaLascoux J. et Weil P., Logiques d'Etats et immigrations, Paris, Kimé, 1992, pp. 13-57.

8. Siméant J., La cause des sans-papiers, Paris, Presse de Sciences Po, 1998, p. 305.

9. Foucault M., « La gouvernementalité », Dits et écrits T. III, Paris, Gallimard, 1994, pp. 635-657.

10. Cette étude s'appuie sur les documents versés par le ministère de l'Intérieur au Centre des Archives Contemporaines de Fontainebleau (séries 19990137 art. 5. « Mise en place de centres de rétention administrative des étrangers en situation irrégulière. 1985-1989») ; ainsi que sur les archives internes de l'association Cimade. Je tiens ici à remercier les membres de la Cimade-DER Paris, notamment Laurent Giovannoni et Jérôme Martinez, qui ont bien voulu me confier leur documentation.

11. Ce centre est encore en activité aujourd'hui. Voir Panzani A., Une prison clandestine de la police française: Arenc, Paris, Maspéro, 1975 ; également Spire A., "Rétention: une indignation oubliée », Plein droit, 2001, n50, pp. 20-22 ; et Lochak D., « La place du droit dans la radicalisation de la politique d'immigration.», Communication à la table ronde "La radicalisation politique ",VII ${ }^{\text {ème }}$ congrès de l'AFSP, Lille, septembre 2002.

12. Voir Israël L., « Faire émerger le droit des étrangers en le contestant. Ou l'histoire paradoxale des premières années du GISTI », Politix, n62, 2003, pp. 115-143.

13. . Voir les comptes-rendus de la « disparition » de Mohamed Chérif dans L'Humanité le 19 avril 1975 ; de Mohamed Benia dans Libération le 25 avril 1975, ou encore de Salah Berrebouh dans Le Monde le 7 mai 1975.

14. Foucault M., Surveiller et punir, Paris, Gallimard, 1974 ; voir également Bietlot M., « Du disciplinaire au sécuritaire : de la prison au centre fermé », Multitudes, n¹1, hiver 2003, pp. 57-66. 15. Extraits de la circulaire $n^{\circ} 83-229$ signée Gaston Deferre du 4 octobre 1983, et du compterendu du Secrétariat général du gouvernement daté du 28 décembre 1983 des réunions interministérielles des 24 novembre et 22 décembre 1983, CAC 19990137 art. 5., carton 06/07 1985.

16. Goffman E., Stigmates. Les usages sociaux du handicap, Paris, Minuit, 1968, chap. 3.

17. Ibid, p. 78 . 
18. Sur tous ces aspects, voir Jobard F., Bavures policières? La force publique et ses usages, Paris, La Découverte, 2002.

19. Notion empruntée par G. Engbersen à E. Hugues, in Engbersen G, «The Unknown City », Berkeley Journal of Sociology, vol. 40, 1995-96, p. 104.

20. Voir Engbersen G., « The Unknown City », op. cit.

21. Voir les références aux travaux de Simmel sur le secret in Engbersen, «The Unknown City », op. cit.

22. Sur la production d'information comme activité de mise en forme, voir Latour B. et Woolgar S., La vie de laboratoire, Paris, La Découverte, 1988.

23. Bietlot M., « Du disciplinaire au sécuritaire », op. cit.

24. Compte-rendu des réunions interministérielles des 24 novembre et 22 décembre 1983, op. cit.

25. Spire A., " De l'étranger à l'immigré. La magie sociale d'une catégorie statistique ", Actes de la Recherche en Sciences Sociales, 1999, n¹29, pp. 50-56.

26. Compte-rendu des réunions interministérielles des 24 novembre et 22 décembre 1983, op. cit. 27. On reviendra à plusieurs reprises sur cet exemple, qui a fait l'objet d'une étude particulière, tant sur les archives internes de la Cimade qu'aux archives nationales.

28. Nous utilisons ici le terme dans le sens que lui donne Goffman. Voir Goffman A., Asiles, op. cit., p. 59.

29. Article $5 \mathrm{du}$ règlement interne du centre du Mesnil-Amelot, source : Cimade.

30. Voir Bosc A. "Le Mesnil-Amelot»: une journée ordinaire dans un centre de rétention ", Causes Communes, $1995, \mathrm{n}^{\circ} 1$, pp. 6-8.

31. Pour une typologie des «identités de papier» en fonction de la relation à l'Etat qu'ils attestent, voir Torpey J., The Invention of the Passport. Surveillance, Citizenship and the State, Cambridge, Cambridge UP, 2000, pp. 158 et suivantes.

32. Cet usage des « documents " comme attestations est bien mis en évidence dans les situations - fréquentes - dans lesquelles les gendarmes ne disposent pas des papiers de l'étranger. Il est alors présenté au consulat dont il est le ressortissant présumé, pour y subir un interrogatoire contrôlant ses connaissances quand à son pays d'origine supposé. Le résultat le plus fréquent est le refus du laisser-passer consulaire: dans ce cas précisément, l'épreuve de vérité ne peut accéder à la valeur incontestable du document administratif. L'étranger, parce qu'il est capable d'erreur ou de dissimulation, n'est pas une source d'information fiable. Pour des exemples voir Hamel C., Lemoine D., Rendez-vous au 35bis. L'étranger, le juge et l'ethnologue, Paris, Editions de l'Aube, 1997.

33. Voir notamment Noiriel G., Réfugiés et sans-papiers, Paris, Pluriel, 2000, également Piazza P., Histoire de la carte nationale d'identité, Paris, Odile Jacob, 2004.

34. Sur ce point voir Bauman Z., Le coût humain de la mondialisation, Paris, Hachette, 1999, pp. 27 et suivantes.

35. Goffman E., Asiles, op. cit.

36. Bernardot M., «Le pays aux mille et un camps. Approche socio-historique des espaces d'internement en France au XX ${ }^{\text {ème }}$ siècle ", Cahiers du Cériem, n 10, décembre 2002, pp. 57-76.

37. Ces expressions ont été utilisées par Fabien Jobard sur la garde à vue, Bavures policières?, op. cit., pp. 82-83.

38. Ibid., p. 102.

39. Siméant J., La cause des sans-papiers, op. cit., p. 305 et suivantes.

40. Note du contrôleur des armées Carcel sur le fonctionnement du centre du Mesnil-Amelot adressée au ministère de la Défense, 10 mars 1989, CAC 1999 0137/5/ « Mission : CRA 198916846 », p. 4.

41. Siméant J., op. cit., p. 305.

42. Note du contrôleur des armées Carcel, op. cit.

43. Sur ce point, voir Jobard F., op. cit., p. 101. 
44. Nous empruntons l'expression à Goffman, pour qui l'existence de tels transporteurs est cruciale dans les institutions totales en général. Voir Goffman E., Asiles, op. cit., pp. 308 et suivantes.

45. Texte de Pierre Géry daté de 1985, archives internes DER-Cimade.

46. Voir Drahy J., Le droit dans la défense des étrangers par les associations: l'exemple de la Cimade, Mémoire de DEA, Paris II, 2003, pp. 63 et suivantes.

47. Rapport d'un équipier du centre de Sainte Foy lès Lyon publié dans la feuille interne Cimade Info en septembre 1985, archives internes DER-Cimade.

48. Sur ce point, voir Siméant J., "Urgence et développement, professionnalisation et militantisme dans l'humanitaire ", Mots, mars 2001, n65, pp. 28-49.

49. On trouve alors dans les rapports des premières années de lointains échos de l'affaire d'Arenc: "L'information a pu porter sur le centre de rétention lui-même (qu'est-ce que c'est, pourquoi y sont les gens, comment et pour combien de temps), (...) sur la possibilité de recevoir de la visite ou - pour les proches - d'aller rendre visite, de téléphoner ou recevoir des coups de fil... (...) L'information auprès des familles ou amis [a porté] sur le simple fait de dire où était la personne, quand elle partait... Deux ou trois fois, la famille croyait qu'elle était déjà partie on ne sait où, ou même ne savait pas du tout où elle était, ni qu'elle avait été jugée. Auprès des proches, le « déblayage » des possibilités de retour quant à l'avenir s'est avéré également important ", Rapport d'un équipier du centre de Sainte Foy lès Lyon, op. cit, non paginé.

50. Citons les récupérations de bagages effectuées par la Cimade dans des hôtels que les retenus peinent à situer exactement, ou la recherche menée à " Aix, Toulon et Avignon » par un équipier de Marseille pour retrouver la fille d'une Algérienne qui refuse de partir sans elle. Exemples cités dans le rapport partiel de la Cimade pour 1986, archives internes Cimade.

51. Réponse du gestionnaire du centre du Mesnil-Amelot à un questionnaire de l'Inspection Générale de l'Administration, printemps 1989, CAC 1999 0137/5/ « Mission : CRA 198916846 ».

52. Extrait du procès-verbal du Conseil de la Cimade du 19 mai 1984, archives internes Cimade.

53. Sur ce point, voir Drahy J., op. cit., p. 59.

54. Sur ce point, voir Cefaï D., «La construction des problèmes publics. Redéfinitions de situations dans des arènes publiques ", Réseaux, n75, 1996, pp. 45-60.

55. Motion votée à l'issue de la session des équipiers en rétention du 27 janvier 1987, archives internes Cimade.

56. Hirschman A. O., Bonheur privé, action publique, Paris, Fayard, 1983.

57. Lettre de Jean Costil à Marianne Hutzinger du 16 novembre 1989, op.cit.

58. Ibid.

59. Ibid.

60. Elle s'est trouvée récemment mise à exécution en mai 2004, par le retrait total de la Cimade pour une durée d'un mois.

61. Procès-verbal du conseil du 21 septembre 1990, archives internes Cimade.

62. La notion, introduite dans la convention au début des années 1990, a fait l'objet d'une précision supplémentaire avec le décret de mars 2001, qui évoque «l'exercice effectif des droits des étrangers retenus» parmi les missions des équipiers Cimade. Voir Drahy J., op. cit., pp. 63 et suivantes.

63. Compte-rendu de la réunion du 2 mars 2000 à la préfecture de Seine et Marne, archives internes de la DER Paris.

64. Lettre du 3 septembre 2001 adressée par la DER-Paris à la préfecture de Seine et Marne, archives internes de la DER Paris.

65. Lettre adressée à la DER Cimade à la préfecture de Seine et Marne, 15 janvier 1998, Ibid.

66. Lettre adressée par le coordinateur de la DER à la Préfecture de Seine et Marne, 7 janvier 2000, Ibid.

67. Cimade, Centres de rétention administrative. Rapport 2002, Paris, Cimade, 2003, p. 8. 
68. Cimade, Centres de rétention administrative. Rapport 2000, Paris, Cimade, 2001, p. 128.

69. Article 35bis de l'ordonnance du 2 novembre 1945 sur l'entrée et le séjour des étrangers modifiée, alinéa VI.

70. La productionde la «psychologie» du retenu comme objet de la sollicitude des gardiens la rapproche ainsi des « réalités de transaction » évoquées par Michel Foucault : « (...) C'est comme la folie, c'est comme la sexualité. C'est ce que j'appellerai des réalités de transaction, c'est-à-dire que dans le jeu précisément et des relations de pouvoir et de ce qui sans cesse leur échappe, c'est de cela que naissent, en quelque sorte à l'interface des gouvernants et des gouvernés, ces figures transactionnelles et transitoires qui, pour n'avoir pas existé de tout temps, n'en sont pas moins réelles (...) ». Voir Foucault M., Naissance de la biopolitique, Cours au Collège de France 1978-1979, Paris, Gallimard/Seuil, 2004, pp. 300-301.

71. Cette association entre l'ouverture tolérée du centre et son contrôle par des dispositifs de sécurité rappelle là encore l'association entre sécurité et liberté chez le dernier Foucault. Voirnotamment Foucault M. Sécurité, territoire, population. Cours au Collège de France 1977-1978, Paris, Gallimard/Seuil, 2004.

\section{RÉSUMÉS}

Parmi les espaces d'internement destinés aux étrangers qui se multiplient aujourd'hui en Europe, les centres de rétention administrative présentent la particularité de se trouver directement au contact des sociétés occidentales, mais aussi de leurs espaces publics. Ces centres s'insèrent alors dans un dispositif plus général de détection des étrangers présents irrégulièrement sur le territoire des Etats Schengen. Il doivent à ce titre permettre de prélever sur eux une information favorisant leur éloignement $\mathrm{du}$ territoire ou tout au moins leur «traçabilité ». A travers l'exemple d'un centre de rétention français, cette contribution s'efforce d'analyser l'organisation de ce prélèvement de l'information, mais elle envisage également la manière dont cette organisation est infléchie voire déjouée par la possibilité d'un contact non formalisé et non maîtrisé des étrangers retenus avec l'espace public dont ils ont été retranchés, notamment à travers le transport de l'information par les équipiers de la Cimade présents au centre.

The current development of detention camps for undocumented foreigners in the Schengen area underlines the specificity of « deportation camps », especially designed to manage the population of expelled foreigners formerly present on the territory of a member State. Settled inside European countries, those camps come in addition to the various devices created in order to track and detect undocumented foreigners. In that sense, deportation camps are part of an information-collecting apparatus. Through the example of a French "centre de retention ", this contribution aims to analyse the organisation of that information collect. In this attempt, the impact of informal contacts of the detainees with the French national public sphere, and the role of the Cimade association in their development, shall particularly be examined.

\section{INDEX}

Mots-clés : Camps, Europe, centre de rétention administrative, information 
AUTEUR

NICOLAS FISCHER

Nicolas FISCHER est Doctorant à l'IEP Paris/CEVIPOF, et ATER à l'Université François-Rabelais de Tours. 